\title{
The Performance Measurement of Generalized Sharpe Ratio and Economic Performance Measure: A Hedge Funds Example
}

\author{
Pham Ngoc Van ${ }^{1, *}$, Khoa Dang Duong ${ }^{2}$ \\ ${ }^{1}$ Faculty of Finance and Banking, Industrial University of Ho Chi Minh City, Vietnam \\ ${ }^{2}$ Faculty of Finance and Banking, Ton Duc Thang University, Ho Chi Minh City, Vietnam \\ ${ }^{*}$ Corresponding Author: phamngocvan@iuh.edu.vn
}

Received October 14, 2021; Revised December 7, 2021; Accepted December 23, 2021

\section{Cite This Paper in the following Citation Styles}

(a): [1] Pham Ngoc Van, Khoa Dang Duong, "The Performance Measurement of Generalized Sharpe Ratio and Economic Performance Measure: A Hedge Funds Example," Universal Journal of Accounting and Finance, Vol. 10, No. 1, pp. 124-130, 2022. DOI: 10.13189/ujaf.2022.100113.

(b): Pham Ngoc Van, Khoa Dang Duong (2022). The Performance Measurement of Generalized Sharpe Ratio and Economic Performance Measure: A Hedge Funds Example. Universal Journal of Accounting and Finance, 10(1), 124-130. DOI: 10.13189/ujaf.2022.100113.

Copyright $\bigcirc 2022$ by authors, all rights reserved. Authors agree that this article remains permanently open access under the terms of the Creative Commons Attribution License 4.0 International License

\begin{abstract}
Prior literature documents that the Sharpe ratio (SR) generates biases in performance evaluation if returns distribution deviates from normal distribution because SR is derived under the mean-variance model with the strict assumption of either quadratic preferences or customarily distributed returns. When the return distributions deviate from normality, it may lead to unreasonable results. Therefore, this study examines which performance measurement approaches are efficient for non-normality on the distribution of asset returns. We collect monthly returns of 14 Credit Suisse (CS) hedges fund indexes from April 1994 to June 2021. The hedge fund index returns exhibit high negative skewness or high positive kurtosis, implying non-normal distribution. Then, we employ the Sharpe ratio (SR) and two performance measures, which extend the Sharpe ratio, the generalized Sharpe ratio (GSR), and the economic performance measure (EPM), to evaluate the performances of hedge funds. In addition, both the nonparametric and parametric estimation methods of the GSR and the EPM are utilized. Our findings indicate that the nonparametric GSR and the nonparametric EPM produce more similar rankings than the SR. Among the three parametric estimation methods of the GSR and EPM, only the method proposed by [1] produces similar rankings with the nonparametric GSR and the nonparametric EPM. Finally, our study contributes the practical approach for fund managers to evaluate their fund performance efficiently.
\end{abstract}

Keywords Sharpe Ratio, EPM, GSR, Performance Evaluation, Hedge Funds, Nonparametric, Parametric

JEL Classification Codes: G2, G11, G15

\section{Introduction}

The Sharpe ratio (SR) is a popularly used performance measure of portfolio management. However, the SR has some limitations and shortcomings that have been extensively discussed in the literature. It is derived under the mean-variance model with the strict assumption of either quadratic preferences or customarily distributed returns. When the return distributions deviate from normality, it may lead to unreasonable results $[3,4,19]$. For instance, Hodges[12] demonstrates the Sharpe ratio paradox by comparing two similar distributions, A and B. Hodges[12] pointed out distribution A was performed better than distribution $\mathrm{B}$; however, the standard deviation of distribution $\mathrm{A}$ increased more significant percentage than the increase of mean of distribution $\mathrm{B}$. That is the reason why Hodges[12] comes up that the Sharpe ratio results in wrong rankings in the Sharpe ratio paradox.

In addition, the Sharpe ratio can be manipulated, so its role as a performance measure is doubtful (Leland[16]; Goetzmann et al.[8]; Goetzmann et al.[9]). Evaluation 
strategies from [8] can alter the shape of returns distribution. For instance, Henriksson and Merton [11] argued that applying the Sharpe ratio had been restricted and caused performance valuation problems because of non-linear payoffs. Furthermore, the Sharpe ratio generates the wrong valuation when the return distribution has deviated from normality [17]. The study of [17] also proved that flattening returns can increase the Sharpe ratio for a long time. As a result, the Sharpe ratio cannot perform well for portfolio performance measures.

There are two methodologies to extend the Sharpe ratio, which go beyond the mean and variance to mitigate the shortcomings. Hodges [12] and Zakamouline and Koekebakker [21] propose a new performance measure called the generalized Sharpe ratio (GSR), based on the expected utility theory of portfolio choice. Moreover, Homm and Pigorsch[13] constructed another new performance measure called the economic performance measure (EPM) by replacing the standard deviation of the Sharpe ratio with a riskiness index [2]. Both performance measures are a kind of generalized Sharpe ratio because one reduces to the Sharpe ratio. The other is equivalent to the Sharpe ratio ranking when the return distribution converges to a normal distribution.

To examine the differences between the SR and the GSR, or the EPM on performance evaluation, Zakamouline and Koekebakker[21] and Homm and Pigorsch[13] separately evaluate performances of hedge funds because the hedge fund returns often exhibit high negative skewness and high positive kurtosis. This paper collects monthly returns of 14 Credit Suisse (CS) hedges fund indexes from April 1994 to June 2021. We put all three performance measures in evaluating the performances of hedge funds to examine the differences among the three measures. Theoretically, the GSR and the EPM are expected to produce more similar performance rankings than the SR.

Prior literature calculates parametric and nonparametric estimation methods of performance measures. By assuming that the return follows a Normal Inverse Gaussian (NIG) distribution, the study of [13] and [21] derive a different closed-form formula of the parametric estimators. Another closed-form formula for a parametric estimator is also derived by [1], using the Taylor approximation. All three estimators consider the first four moments of distribution in computing performance measures. The nonparametric estimations are based on numerical methods and consider all moments of the distribution. The parametric methods are easier to compute, but they are only approximations of the nonparametric estimations. In applying the riskiness index of [2] for finding optimal hedge ratio, [21] find out that various non-normal distributions could have different riskiness even if they have the same first four distribution moments. Thus, it is interesting to examine if the parametric methods can produce similar results to the nonparametric.

This paper contributes to the literature from two prospective. First, we compare two performance measures that generalize the Sharpe ratio, the GSR and the EPM, by their ranking performances of hedge funds. We employ both the parametric and the nonparametric methods of the GSR and the EPM. The GSR(P-A) is the parametric estimate of the generalized Sharpe ratio (GSR) proposed by [1]. The GSR(NP) is the nonparametric estimate of GSR proposed by [21]. The studies of [2] and [13] introduced the $\operatorname{EPM}(\mathrm{P})$ and $\operatorname{EPM}(\mathrm{NP})$, respectively, as parametric and nonparametric estimates of the economic performance measure. Second, using the same data, we also examine the estimates of the performance measures of the parametric and nonparametric estimation methods to see how close the parametric estimates are to the nonparametric. The results indicate that the nonparametric GSR and the nonparametric EPM produce more similar rankings than the SR. Among the three parametric estimation methods of the GSR and EPM, only the method proposed by [1] produces similar rankings with the nonparametric GSR and the nonparametric EPM.

The detail of this chapter is organized as follows. Section 2 reviews the two methodologies for extending the Sharpe ratio. The performance measurements of hedge funds are presented in Section 3. The final section is the conclusion.

\section{Literature Review}

Two methodologies that extend the Sharpe ratio are discussed here. One methodology is based on the expected utility theory of portfolio choice, while the other uses the reward-to-risk framework.

\subsection{The Generalized Sharpe Ratio (GSR)}

Markowitz's portfolio theory based on a mean-variance model indicates that the investors always choose the optimal risky portfolio with the highest Sharpe ratio within a feasible set. Thus, the Sharpe ratio is a natural definition of a performance measure. Also, the mean-variance model assumes that the investor has negative exponential utility and that the risky asset returns are normally distributed. The study of [12] points out that in the mean-variance model with one free asset and one risky portfolio, the maximum utility of investors is given by:

$$
\mathrm{E}\left(U^{*}\right)=-e^{-\mathrm{SR}^{2} / 2}
$$

where SR is the Sharpe ratio of the risky portfolio. Thus, the higher the Sharpe ratio of the risky portfolio, the higher the level of maximum expected utility that the investor can receive.

To go beyond the mean-variance model and generalize the Sharpe ratio, [12] conjectures that the relationship between the maximum utility of investors and the generalized Sharpe ratio (GSR) is:

$$
\mathrm{E}\left(U^{*}\right)=-e^{-\mathrm{GSR}^{2} / 2},
$$


For any return distribution of the risky portfolio. Thus, the GSR can be computed using:

$$
\mathrm{GSR}=\sqrt{-2 \log \left(-\mathrm{E}\left(U^{*}\right)\right)}
$$

by first finding out the maximum expected utility.

To determine the maximum expected utility for any return distribution, Zakamouline and Koekebakker [21] suggest a numerical optimization method of the maximizing problem

$$
\max \mathrm{E}\left(a-e^{-\lambda a\left(r_{p}-r_{f}\right)}\right)
$$

where " $a$ " is the decision variable, $\lambda$ is the negative exponential utility function parameter, $r_{p}$ is the rate of return of the risky portfolio, and $r_{f}$ is the risk-free rate. This method of finding the maximum expected utility and calculating the GSR is referred to as the nonparametric estimation and is called GSR(NP).

To apply the GSR without using the numerical method, [21] derive a GSR formula as a way of parametric estimation by assuming that the portfolio return follows a Normal Inverse Gaussian (NIG) distribution:

$\mathrm{f}\left(r_{p} ; \alpha, \beta, \eta, \delta\right)=\frac{\alpha \delta e^{\delta \varphi+\beta\left(r_{p}-\eta\right)}}{\pi \sqrt{\delta^{2}+\left(r_{p}-\eta\right)^{2}}} K_{1}\left(\alpha \sqrt{\delta^{2}+\left(r_{p}-\eta\right)^{2}}\right)$

where

$$
\begin{gathered}
\varphi=\sqrt{\alpha^{2}-\beta^{2}}, \\
K_{1}(x)=\frac{1}{2} \int_{0}^{\infty} e^{-\left(\frac{1}{2}\right) x\left(z+z^{-1}\right)} d z,
\end{gathered}
$$

and $\alpha, \beta, \eta$, and $\delta$ are parameters. These parameters are linked to the mean, variance, skewness, and kurtosis of the NIG distribution as follows:

$$
\begin{gathered}
\mathrm{E}\left(r_{p}\right)=\mu=\eta+\delta \frac{\beta}{\varphi}, \operatorname{Var}\left(r_{p}\right)=\sigma^{2}=\delta \frac{\alpha^{2}}{\varphi^{3}}, \\
\operatorname{Skew}\left(r_{p}\right)=\chi=3 \frac{\beta}{\alpha \sqrt{\delta \varphi}}, \operatorname{Kurt}\left(r_{p}\right)=\kappa=3+\frac{3}{\delta \varphi}(1+ \\
\left.4\left(\frac{\beta}{\alpha}\right)^{2}\right) .
\end{gathered}
$$

From these equations, we can derive:

$$
\begin{gathered}
\alpha=\frac{3 \sqrt{3 \kappa-4 \chi^{2}-9}}{\sigma^{2}\left(3 \kappa-5 \chi^{2}-9\right)}, \beta=\frac{3 \chi}{\sigma\left(3 \kappa-5 \chi^{2}-9\right)}, \eta=\mu-\frac{3 \chi \sigma}{\left(3 \kappa-4 \chi^{2}-9\right)}, \\
\text { and } \delta=\frac{3 \sigma^{2} \sqrt{3 \kappa-5 \chi^{2}-9}}{\left(3 \kappa-4 \chi^{2}-9\right)}
\end{gathered}
$$

However, to get meaningful parameters $\alpha$ and $\delta$, the following condition must be satisfied:

$$
\kappa>3+\frac{5}{3} \chi^{2}
$$

The formula of the parametric estimation of GSR is given by:

$$
\sqrt{2\left(\lambda a^{*}\left(\eta-r_{f}\right)-\delta\left(\varphi-\sqrt{\alpha^{2}-\left(\beta-\lambda a^{*}\right)^{2}}\right)\right.}
$$

where

$$
a^{*}=\frac{1}{\lambda}\left(\beta+\frac{\alpha\left(\eta-r_{f}\right)}{\sqrt{\delta^{2}+\left(\eta-r_{f}\right)^{2}}}\right)
$$

Thus, to implement the parametric estimation, we simply estimate the first four moments of the return distribution and use them to compute the four parameters of the NIG distribution and the GSR. This estimation method is called GSR(P-Z). The findings from [21] show that GSR can resolve Sharpe ratio paradoxes and reveal the proper performance of portfolios with modified Sharpe ratios.

Another parametric method to estimate the GSR was developed by Alexander [1] and is called GSR(P-A). She used the Taylor expansion of the expected utility to get the certainty equivalent of the portfolio investment and obtain the maximum expected utility by using the approximating method by [17]. The formula is as follows:

$$
\sqrt{\left(\frac{\mu-r_{f}}{\sigma}\right)^{2}+\frac{\chi\left(\frac{\mu-r_{f}}{\sigma}\right)^{3}}{3}-(\kappa-3)\left(\frac{\mu-r_{f}}{\sigma}\right)^{4} / 12}
$$

This formula provides the same result to the Sharpe ratio when the skewness equals 0 , and the kurtosis equals 3 . It also indicates that negative skewness and high kurtosis have the effect of decreasing the GSR. The findings from [13] show that the EPM penalizes investments with significant excess kurtosis (or negative skewness). However, the Sharpe ratio ignores these characteristics

\subsection{The AS Index of Riskiness and the EPM}

The Sharpe ratio is a typical reward-to-risk performance measure. However, it is derived under the mean-variance framework. There, the standard deviation is a legitimate risk measure. The standard deviation is not a good measure under general conditions. Homm and Pigorsch[13] proposed a new performance measure by replacing the standard deviation with the AS index of riskiness. This performance measure is the economic performance measure (EPM).

The AS index is derived based on the two fundamental axioms, duality and positive homogeneity. The duality requires the risk index that reflects how less risk-averse individuals accept risky assets. Thus, it satisfies monotonicity concerning second-order stochastic dominance (SSD). SSD is an essential property for risk measurement. If portfolio A dominates portfolio B in terms of SSD and we know that all risk-averse investors prefer A to $\mathrm{B}$, a risk measure with monotonicity will indicate that portfolio A is less risky than Portfolio B. Standard deviation, semi-standard deviation, value at risk, and expected shortfall violate the monotonicity as a risk measure.

Aumann and Serrano[2] defined the economic index of riskiness for a risky asset as the reciprocal of an individual's positive risk aversion parameter with constant absolute risk aversion (CARA), who is indifferent between taking and not taking the risky asset.

In Homm and Pigorsch[13], the EPM is defined as: 


$$
\mathrm{EPM}=\frac{\mathrm{E}(\tilde{r})}{\mathrm{AS}(\tilde{r})},
$$

Where $\mathrm{E}(\tilde{r})$ is the expected excess return of an investment portfolio, and $\operatorname{AS}(\tilde{r})$ is the $\mathrm{AS}$ index of the random excess return. Homm and Pigorsch[13] demonstrated that the EPM has the favorable monotonicity property concerning the first-order and second-order stochastic dominance. This property is not held by most other performance measures [13].

In applying the EPM, the subtle part estimates the AS index. According to Aumann and Serrano[2], we can obtain the index by solving $\operatorname{AS}(\tilde{r})$ in the Equation:

$$
\mathrm{E}\left(\mathrm{e}^{-\frac{\widetilde{r}}{\mathrm{AS}(\widetilde{r})}}\right)=1
$$

We can apply Equation (10) and solve the AS index without assuming any distribution function given an empirical or simulated distribution. Equation (10) is a nonparametric estimation method. The EPM estimated by this method is referred to as EPM(NP).

The EPM can also be regarded as a generalized Sharpe measure because the EPM equals two times the squared Sharpe ratio under the normality. Thus, the EPM is equivalent to the Sharpe measure ranking under normality.

By also assuming a NIG distribution, Homm and Pigorsch[13] derived the AS index and the EPM as follows:

$$
\begin{aligned}
& \mathrm{AS}=\left(3 \kappa\left(\mu-r_{f}\right)-4\left(\mu-r_{f}\right) \chi^{2}-6 \chi \sigma+\frac{9 \sigma^{2}}{\left(\mu-r_{f}\right)}\right) / 18 \\
& \text { And } \\
& \qquad \mathrm{EPM}=18\left(\mu-r_{f}\right) /\left(3 \kappa\left(\mu-r_{f}\right)-4\left(\mu-r_{f}\right) \chi^{2}-\right. \\
& \left.6 \chi \sigma+\frac{9 \sigma^{2}}{\left(\mu-r_{f}\right)}\right)
\end{aligned}
$$

Equation 12 demonstrates a parametric estimation method, and it is called EPM (P). The formula indicates that positive skewness increases the performance measure, but high kurtosis decreases it.

\section{Data and Research Methodology}

In this section, we use the SR, the GSR, and the EPM to evaluate the performances of hedge funds. The parametric and the nonparametric methods of the GSR and the EPM are employed. The return data of hedge funds is similar to the data used in Zakamouline and Koekebakker[21]. We use the monthly returns of 14 Credit Suisse (CS) hedges fund indexes (including the main index) which track almost every primary style of hedge fund management. There is one index excluded because of its negative mean return. The sample period is from April 1994 to June 2021. The three-month US T-bill rate is used as the risk-free rate proxy. All performance measures calculated here are based on the excess monthly returns. ${ }^{1}$

\section{Empirical Results}

Table 1 reports the first four moments of the hedge fund index returns. As we can see from the table, 10 out of 13 indexes exhibit negative skewness, and only one index does not have excess kurtosis. Thus, even we use much more samples, we can still find style facts of the hedge fund returns.

Table 1. Return statistics of hedge fund indexes

\begin{tabular}{ccccc}
\hline $\begin{array}{c}\text { Hedge fund } \\
\text { index }\end{array}$ & Mean & Std & Skewness & Kurtosis \\
\hline $\begin{array}{c}\text { All Hedge } \\
\text { Index }\end{array}$ & 0.0048 & 0.0202 & -0.2312 & 6.1472 \\
$\begin{array}{c}\text { Convertible } \\
\text { Arbitrage }\end{array}$ & 0.0056 & 0.0190 & -2.6322 & 19.9911 \\
$\begin{array}{c}\text { Emerging } \\
\text { Markets }\end{array}$ & 0.0062 & 0.0399 & -0.8324 & 9.3362 \\
$\begin{array}{c}\text { Equity Market } \\
\text { Neutral }\end{array}$ & 0.0043 & 0.0280 & -12.4646 & 184.0371 \\
$\begin{array}{c}\text { Event Driven } \\
\text { Event Driven } \\
\text { Distressed }\end{array}$ & 0.0068 & 0.0180 & -2.0559 & 12.4207 \\
Event Driven & 0.0064 & 0.0183 & -2.0784 & 13.9583 \\
$\begin{array}{c}\text { Multi-Strategy } \\
\text { Event Driven }\end{array}$ & 0.0048 & 0.0117 & -0.8925 & 7.3304 \\
$\begin{array}{c}\text { Risk Arbitrage } \\
\text { Fixed Income } \\
\text { Arbitrage }\end{array}$ & 0.0045 & 0.0154 & -4.7704 & 39.3008 \\
$\begin{array}{c}\text { Global Macro } \\
\text { Long/Short } \\
\quad \text { Equity }\end{array}$ & 0.0091 & 0.0261 & 0.1650 & 7.7687 \\
$\begin{array}{c}\text { Managed } \\
\text { Futures }\end{array}$ & 0.0052 & 0.0340 & 0.0176 & 2.8792 \\
Multi-Strategy & 0.0064 & 0.0147 & -1.7219 & 9.6226 \\
\hline
\end{tabular}

(Source: Credit Suisse hedges fund indexes, 2021)

Table 2 lists performance measures of the hedge fund indexes. First, we examine how well the parametric methods approximate the nonparametric estimations. The GSR(P-Z) produces relatively large numbers, which are very different from those of the GSR(NP). It does not have any results in three hedge fund indexes because the condition for the meaningful parameters is violated. Thus, we exclude the GSR(P-Z) for further analysis in the following section. The EPM(P) does not approximate well to the EPM(NP). Except for one index, the EPM(P) gives relatively small numbers than the EPM(NP). This finding is consistent with $[3,9,10,14,18]$. Only the GSR(P-A) approximates the GSR(NP) well for most cases. Table 3 reports the rankings based on the SR, the $\operatorname{EPM}(\mathrm{NP})$, the GSR(NP), the GSR(P-A), and the EPM(P). The EPM(P) produces very different rankings from others because of its poor approximation. Thus, we suggest that, for performance evaluation, the GSR(P-Z) and the EPM(P) should be used with caution. Our results align with $[4,5,6$, $15,20]$ 
Table 2. Performance measures of hedge fund indexes

\begin{tabular}{|c|c|c|c|c|c|c|}
\hline Hedge fund index & SR & $\operatorname{EPM}(\mathbf{P})$ & EPM(NP) & GSR(NP) & GSR(P-A) & GSR(P-Z) \\
\hline All Hedge Index & 0.2400 & 0.0086 & 0.1057 & 0.2367 & 0.2360 & 2.2173 \\
\hline $\begin{array}{l}\text { Convertible } \\
\text { Arbitrage }\end{array}$ & 0.2972 & 0.0020 & 0.1091 & 0.2636 & 0.2345 & 5.2444 \\
\hline Emerging Markets & 0.1549 & 0.0052 & 0.0426 & 0.1513 & 0.1507 & 1.5514 \\
\hline $\begin{array}{c}\text { Equity Market } \\
\text { Neutral }\end{array}$ & 0.1535 & 0.0001 & 0.0223 & 0.1241 & 0.0256 & NA \\
\hline Event Driven & 0.3770 & 0.0042 & 0.1756 & 0.3350 & 0.3012 & 8.3420 \\
\hline $\begin{array}{l}\text { Event Driven } \\
\text { Distressed }\end{array}$ & 0.4215 & 0.0042 & 0.2037 & 0.3676 & 0.3143 & 6.7204 \\
\hline $\begin{array}{l}\text { Event Driven } \\
\text { Multi-Strategy }\end{array}$ & 0.3250 & 0.0057 & 0.1488 & 0.2992 & 0.2859 & 7.0406 \\
\hline $\begin{array}{c}\text { Event Driven Risk } \\
\text { Arbitrage }\end{array}$ & 0.4118 & 0.0066 & 0.2444 & 0.3839 & 0.3731 & 6.4033 \\
\hline $\begin{array}{l}\text { Fixed Income } \\
\text { Arbitrage }\end{array}$ & 0.2891 & 0.0007 & 0.0864 & 0.2420 & 0.1591 & NA \\
\hline Global Macro & 0.3491 & 0.0111 & 0.2145 & 0.3450 & 0.3442 & 1.9264 \\
\hline Long/Short Equity & 0.2886 & 0.0116 & 0.1525 & 0.2858 & 0.2850 & 1.7381 \\
\hline Managed Futures & 0.1519 & 0.0602 & 0.0461 & 0.1523 & 0.1520 & NA \\
\hline Multi-Strategy & 0.4406 & 0.0057 & 0.2465 & 0.3930 & 0.3547 & 10.9812 \\
\hline
\end{tabular}

(Source: Author's calculation based on data from Credit Suisse hedges fund indexes, 2021)

Notes: The SR stands for the Sharpe ratio. The GSR(P-A) is the parametric estimate of the generalized Sharpe ratio (GSR) proposed by Alexander[1]. The GSR(NP) is the nonparametric estimate of GSR. The EPM(P) and EPM(NP) are the parametric and nonparametric estimates of the economic performance measure

Notes: The Mean, Std, Skewness, and Kurtosis are the first fourth moments of the distributions.

Table 3. Rankings of hedge fund indexes by different measures and methods

\begin{tabular}{|c|c|c|c|c|c|}
\hline Hedge fund index & SR & EPM(NP) & GSR(NP) & GSR(P-A) & $\operatorname{EPM}(\mathbf{P})$ \\
\hline All Hedge Index & 10 & 9 & 10 & 8 & 4 \\
\hline Convertible Arbitrage & 7 & 8 & 8 & 9 & 11 \\
\hline Emerging Markets & 11 & 12 & 12 & 12 & 8 \\
\hline Equity Market Neutral & 12 & 13 & 13 & 13 & 13 \\
\hline Event-Driven & 4 & 5 & 5 & 5 & 9 \\
\hline Event-Driven Distressed & 2 & 4 & 3 & 4 & 10 \\
\hline $\begin{array}{l}\text { Event-Driven } \\
\text { Multi-Strategy }\end{array}$ & 6 & 7 & 6 & 6 & 7 \\
\hline $\begin{array}{c}\text { Event-Driven Risk } \\
\text { Arbitrage }\end{array}$ & 3 & 2 & 2 & 1 & 5 \\
\hline Fixed Income Arbitrage & 8 & 10 & 9 & 10 & 12 \\
\hline Global Macro & 5 & 3 & 4 & 3 & 3 \\
\hline Long/Short Equity & 9 & 6 & 7 & 7 & 2 \\
\hline Managed Futures & 13 & 11 & 11 & 11 & 1 \\
\hline Multi-Strategy & 1 & 1 & 1 & 2 & 6 \\
\hline
\end{tabular}

(Source: Author's calculation based on data from Credit Suisse hedges fund indexes, 2021)

Notes: The SR stands for the Sharpe ratio. The GSR(P-A) is the parametric estimate of the generalized Sharpe ratio (GSR) proposed by Alexander[1]. The GSR(NP) is the nonparametric estimate of GSR. The EPM(P) and $\operatorname{EPM}(\mathrm{NP})$ are the parametric and nonparametric estimates of the economic performance measure.

Finally, we list the rank correlation (Kendall's $\tau$ ) for the rankings Table 4. From the rank correlation, we can examine how close the rankings produced by the different measures. From the table, we can identify a relatively high correlation among the EPM(NP), the GSR(NP), and the GSR(P-A). Then, the SR has a little bit less correlation with the above three. The rank correlation coefficients are
0.66, 0.72, and 0.63. The $\operatorname{EPM}(\mathrm{P})$ has the lowest rank correlation. Thus, we can conclude that the EPN(NP), the GSR(NP), and the GSR(P-A) produce more similar rankings than the SR. Only the GSR(P-A) approximates well for the three parametric estimation methods. Our findings are consistent with $[7,8,16,17,18]$ 
Table 4. Rank correlation for the rankings of hedge fund indexes

\begin{tabular}{ccccc}
\hline & SR & EPM(NP) & GSR(NP) & GSR(P-A) \\
\hline SR & 1 & & & \\
EPM(NP) & 0.66 & 1 & 1 & 1 \\
GSR(NP) & 0.72 & 0.78 & 0.77 & 0.20 \\
GSR(P-A) & 0.63 & 0.78 & 0.11 & 1 \\
EPM(P) & -0.03 & 0.18 & & 1 \\
\hline
\end{tabular}

(Source: Author's calculation based on data from Credit Suisse hedges fund indexes, 2021)

Notes: The SR stands for the Sharpe ratio. The GSR(P-A) is the parametric estimate of the generalized Sharpe ratio (GSR) proposed by Alexander[1]. The GSR(NP) is the nonparametric estimate of GSR. The EPM(P) and EPM(NP) are the parametric and nonparametric estimates of the economic performance measure.

\section{Conclusions}

Although the Sharpe ratio is a popular performance measurement, it has various limitations. SR is based on the mean-variance model with the strict assumption of either quadratic preferences or customarily distributed returns. When return distributions deviate from normality, it may lead to unreasonable results. Therefore, this paper studies the performance measurement of two new performance measures, the GSR and EPM, which extend the Sharpe ratio. These two measures go beyond the mean-variance framework. Thus, they benefit from evaluating the return distributions that deviate from normal distributions. We use the hedge fund returns for this empirical study because the hedge fund index returns exhibit high negative skewness or high positive kurtosis, implying non-normal distribution. Our empirical findings indicate that the nonparametric GSR and the nonparametric EPM produce a more similar ranking than the SR. We also examine three parametric methods which approximate the nonparametric estimations of the GSR or the EPM. The results show that only the method proposed by Alexander[1] approximates well and produces similar rankings to the nonparametric GSR and the nonparametric EPM. Finally, our study contributes the practical approach for fund managers to evaluate their fund performance efficiently.

\section{Acknowledgments}

The authors thank reviewers from Mathematics and statistics Journal, discussants from The 3rd International Conference on Finance and Economics 2016 (ICFE 2016) for constructive feedback, which help us complete this paper.

\section{REFERENCES}

[1] Alexander, C. (2008). Market risk analysis, quantitative methods in finance. Chichester, England: Wiley.

[2] Aumann, R., \& Serrano, R. (2008). An Economic Index of Riskiness. Journal of Political Economy, 116(5), 810-836.

\section{DOI:10.1086/591947}

[3] Darolles, S., Gouriéroux, C., \& Jasiak, J. (2009). L-performance with an application to hedge funds. Journal of Empirical Finance, 16(4), 671-685. DOI: 10.1016/j.jem pfin.2009.05.003

[4] De Souza, C., \& Gokcan, S. (2004). Hedge fund investing: A quantitative approach to hedge fund manager selection and de-selection. The Journal of Wealth Management, 6(4), 52-73. DOI:10.3905/jwm.2004.391058

[5] Eling, M. (2006). Performance measurement of hedge funds using data envelopment analysis. Financial Markets and Portfolio Management, 20(4), 442-471. DOI:10.1007/ s11408-006-0032-4

[6] Eling, M., \& Schuhmacher, F. (2007). Does the choice of performance measure influence the evaluation of hedge funds? Journal of Banking \& Finance, 31(9), 2632-2647. DOI:10.1016/j.jbankfin.2006.09.015

[7] Farinelli, S., Ferreira, M., Rossello, D., Thoeny, M., \& Tibiletti, L. (2008). Beyond Sharpe ratio: Optimal asset allocation using different performance ratios. Journal of Banking \& Finance, 32(10), 2057-2063.DOI:10.1016/j.jba nkfin.2007.12.026

[8] Goetzmann, W. N., Jr., J. E., Spiegel, M. I., \& Welch, I. (2004). Sharpening Sharpe Ratios. SSRN Electronic Journal SSRN Journal. DOI:10.2139/ssrn.302815

[9] Goetzmann, W., Ingersoll, J., Spiegel, M., \& Welch, I. (2007). Portfolio Performance Manipulation and Manipulation-proof Performance Measures. Review of Financial Studies, 20(5), 1503-1546.DOI:10.1093/rfs/hhm 025

[10] Harris, R. D., \& Mazibas, M. (2013). Dynamic hedge fund portfolio construction: A semi-parametric approach. Journal of Banking \& Finance, 37(1), 139-149. DOI:10.1016/j.jbankfin.2012.08.017

[11] Henriksson, R. D., \& Merton, R. C. (1981). On market timing and investment performance. II. Statistical procedures for evaluating forecasting skills. Journal of business, 513-533.

[12] Hodges, S. (1998). A generalization of the Sharpe ratio and its applications to valuation bounds and risk measures. Financial Options Research Centre, Warwick Business School, University of Warwick.

[13] Homm, U., \& Pigorsch, C. (2012). Beyond the Sharpe ratio: An application of the Aumann-Serrano index to performance measurement. Journal of Banking \& Finance, 
36(8), 2274-2284. DOI: 10.1016/j.jbankfin.2012.04.005

[14] Chen, Y.-T., Ho, K.-Y., \& Tzeng, L. Y. (2014). Riskiness-minimizing spot-futures hedge ratio. Journal of Banking \& Finance, 40, 154-164. DOI: 10.1016/j.jbankfin .2013.11.038

[15] Ledoit, O., \& Wolf, M. (2008). Robust performance hypothesis testing with the Sharpe ratio. Journal of Empirical Finance, 15(5), 850-859. DOI:10.1016/j.jempfi n.2008.03.002

[16] Leland, H. E. (1999). Beyond Mean-Variance: Performance Measurement in a Nonsymmetrical World (corrected). Financial Analysts Journal, 55(1), 27-36. DOI:10.2469/faj. v55.n1.2239

[17] Liu, Y., \& Li, Y.-N. (2017). A parametric Sharpe ratio optimization approach for fuzzy portfolio selection problem. Mathematical Problems in Engineering, Article ID
6279859, 17 pages. DOI: 10.1155/2017/6279859

[18] Nguyen-Thi-Thanh, H. (2010). On the consistency of performance measures for hedge funds. Journal of Performance Measurement, 14(2), 1-16.

[19] Pézier, J. (2008) Maximum certain equivalent returns and equivalent performance criteria. ICMA Centre Discussion Papers in Finance

[20] Roslin Lazarus, Stanley Lazarus, Shobhna Gupta (2021). Influence on Corporate Performance by Determinants of Working Capital. Universal Journal of Accounting and Finance, 9(3), 411 - 423. DOI: 10.13189/ujaf.2021.090316.

[21] Zakamouline, V., \& Koekebakker, S. (2009). Portfolio performance evaluation with generalized Sharpe ratios: Beyond the mean and variance. Journal of Banking \& Finance, 33(7), 1242-1254. DOI: 10.1016/j.jbankfin.2009. 01.005 